\title{
Communication during counseling sessions about inhaled corticosteroids at the community pharmacy
}

\author{
Jeanine A Driesenaar' \\ Peter AGM De Smet ${ }^{2,3}$ \\ Rolf van Hulten ${ }^{4,5}$ \\ Litje $\mathrm{Hu}^{\prime}$ \\ Sandra van Dulmen $1,6,7$
}

'NIVEL, Netherlands institute for health services research, Utrecht, the Netherlands; ${ }^{2}$ Department of Clinical Pharmacy, Radboud University Medical Center, Nijmegen, the Netherlands; ${ }^{3} \mathrm{Q}$ Healthcare, Radboud University Medical Center, Nijmegen, the Netherlands; ${ }^{4}$ Division of Pharmacoepidemiology and Clinical Pharmacology, Utrecht University, Utrecht, the Netherlands; ${ }^{5}$ Department of Pharmacotherapy and Pharmaceutical Care,

University of Groningen, Groningen, the Netherlands; ${ }^{6}$ Department of Primary and Community Care, Radboud University Medical Center, Nijmegen, the Netherlands; ${ }^{7}$ Faculty of Health Sciences, University College of Southeast Norway, Drammen, Norway

Correspondence: Jeanine A Driesenaar NIVEL, Netherlands institute for health services research, PO Box 1568 , 3500 BN Utrecht, the Netherlands $\mathrm{Tel}+3$ I 302729707 Email j.driesenaar@nivel.nl
This article was published in the following Dove Press journal:

Patient Preference and Adherence

2 November 2016

Number of times this article has been viewed

Background: Pharmaceutical care is one of the major tasks of pharmacists, which aims to improve patient outcomes. Counseling patients with asthma or chronic obstructive pulmonary disease about their use of inhaled corticosteroids (ICS) might enhance medication adherence and symptom control. Therefore, effective pharmacist-patient communication is very important. In this regard, both affective communication, for handling emotions, and instrumental communication, for exchanging biomedical and lifestyle information, are relevant. Until now, only few studies have explored pharmacist-patient communication, and further insight is needed in this regard. The aim of this study is to investigate how pharmacists and pharmacy technicians communicate about ICS with patients with asthma and/or chronic obstructive pulmonary disease, what topics are discussed by them, and whether pharmacists and pharmacy technicians differ in their communication during counseling sessions.

Methods: Patients aged $\geq 18$ years who had used ICS for at least 1 year and filled at least two ICS prescriptions in the preceding year were recruited through 12 pharmacies. Participants had one counseling session with a pharmacist or a pharmacy technician, which was video-recorded. The process and content of the provider-patient communication were analyzed using the Roter interaction analysis system, adapted to the pharmaceutical setting.

Results: A total of 169 sessions were recorded and analyzed. The communication appeared largely instrumental. Lifestyle, psychosocial issues, and ICS adherence were not discussed in detail. The pharmacists had longer conversations and more affective talk than the pharmacy technicians.

Conclusion: Pharmacists and pharmacy technicians may need to pay more attention to ICS adherence, lifestyle, and psychosocial topics. They differed in their communication; the pharmacists exhibited more affective behavior and discussed medical and therapeutic issues more extensively compared to the pharmacy technicians. Educational courses for pharmacists and pharmacy technicians could focus more on the discussion of adherence, lifestyle, and psychosocial topics with patients.

Keywords: community pharmacy, communication, inhaled corticosteroids, pharmacist-patient interaction, Roter interaction analysis system

\section{Introduction}

According to the Pharmaceutical Care Network Europe definition, "Pharmaceutical care is the pharmacist's contribution to the care of individuals in order to optimize medicines use and improve health outcomes". ${ }^{1}$ The community pharmacist's role has recently been extended from just dispensing to providing guidance in pharmaceutical care. Several pharmaceutical care programs and activities have been developed to 
educate and counsel patients with (chronic) medication use, showing some promising results in improving outcomes such as disease control and medication adherence. ${ }^{2-5}$

Patients with asthma and chronic obstructive pulmonary disease (COPD) could especially benefit from these activities. Around $40 \%$ of them fail to take their inhaled corticosteroids (ICS) as prescribed. ${ }^{6}$ Regular ICS treatment reduces symptoms and the frequency of exacerbations and enhances lung function and quality of life. ${ }^{7,8}$ Taking less medication than prescribed (suboptimal adherence) leads to poor disease control, morbidity, mortality, and high health care costs. ${ }^{6}$ Many patients with asthma $(17 \%-57 \%)$ do indeed have poor disease control, ${ }^{9}$ as shown by the high rates of emergencyroom visits and hospital admissions. ${ }^{10}$

Sufficient and correct knowledge about the disease and medication is needed to achieve better adherence to antiasthma drugs and have better asthma control. ${ }^{11,12}$ But the knowledge among patients with asthma appears suboptimal. ${ }^{13}$ Many patients report taking their medication only when they think they should or when they experience shortness of breath. ${ }^{14}$ In addition, $70 \%$ of patients using ICS apply incorrect inhaler techniques that disenable total absorption of the medication in the lungs. ${ }^{15}$ Improving inhaler technique is likely to improve symptom control. ${ }^{16}$

Clearly, educating patients with asthma and COPD about their disease, medication use, and inhalation technique is of great importance in enhancing ICS adherence and symptom control. This is even more so as patients' potentially modifiable medication beliefs are related to ICS adherence. ${ }^{17-19}$ Patients who have many concerns and misconceptions about ICS (eg, about side effects and drug dependency) are more inclined to be nonadherent. Therefore, health care providers should try to diminish patients' concerns and strengthen their ICS necessity beliefs, ${ }^{17,19}$ by showing empathy and providing reassurance and information about ICS use and adherence. However, medication adherence is hardly addressed at all in clinical encounters, ${ }^{20,21}$ even though Zolnierek and Dimatteo ${ }^{22}$ showed that the odds of patient adherence are 2.16 times higher when a physician communicates effectively.

To perform their new role as communicators, pharmacists and pharmacy technicians receive training in patient education and communication in undergraduate and postgraduate courses. ${ }^{23}$ However, so far, little is known about the extent to which they actually apply these skills in counseling sessions. ${ }^{24}$ Recent research indicates that pharmacists focus mainly on biomedical issues and pay little attention to psychosocial issues. ${ }^{25,26}$

In counseling sessions, pharmacists or technicians can discuss patients' experiences with (chronic) medication, usually in a private consulting room at the community pharmacy. Although some pharmacies do offer counseling sessions to patients about their ICS use, these sessions are not common practice in the Netherlands yet. ${ }^{23,27}$ Furthermore, it is unknown whether pharmacists and technicians are equally able to conduct these sessions. For instance, there are differences in the undergraduate and postgraduate courses for pharmacists and technicians. As technicians have more frequent contacts with patients than pharmacists, ${ }^{28}$ they might be more experienced in patient communication. A study of patients' perceived barriers in communication with their health care provider revealed that patients experience fewer barriers to participation in a visit with a nurse than with a physician. ${ }^{29}$ This might also apply to pharmacists and technicians, with fewer barriers in talks with technicians. However, pharmacists might have a more persuasive communication style by virtue of their position, as they are the managers and often owners of the pharmacy. Getting insight into the differences in the communication content and process might have relevance for session and patient outcomes and might indicate who is most capable of performing these counseling sessions and who might need additional communication training.

The aim of this study was therefore to explore 1) how pharmacists and pharmacy technicians communicate with patients with asthma and/or COPD about ICS during a counseling session (communication process); 2) what topics are discussed during these sessions (communication content); and 3) to what extent pharmacists and technicians differ in their communication. Based on practice guidelines about asthma and COPD, we thereby focus especially on the following communicative aspects: ${ }^{30-32}$ affective communication (eg, showing empathy and giving reassurance), providing information about asthma/COPD and ICS, discussing adherence, inhalation technique, side effects, concerns, and necessity beliefs about ICS, and lifestyle topics (eg, smoking and exercise).

\section{Materials and methods Setting}

This observational study is part of a larger research project examining the communication of community pharmacists and technicians with patients with asthma and/or COPD, which was performed between September 2011 and February 2012. Data were collected with the help of pharmacists belonging to the Utrecht Pharmacy Practice Network for Education and Research (UPPER), and the work was conducted in compliance with the requirements of the UPPER institutional review board of the Department of Pharmacoepidemiology and Clinical Pharmacology. A total 
of 130 community pharmacies were approached via email by UPPER and invited to participate, with 12 pharmacies actually participating. No selection criteria were applied for pharmacies' participation.

Per pharmacy, recruited patients were randomly assigned to an intervention group or a control group. Randomization was performed using a statistical program (Stata Version 12.1), which generated lists consisting of a random series of $0 \mathrm{~s}$ and $1 \mathrm{~s}$. We generated a separate list for each pharmacy. A "0" referred to assignment to the control group, and a "1" referred to assignment to the intervention group. By following the order of the numbers on the list, patients were assigned to the two groups in the sequence of their registration for participation.

Patients in the intervention group had a counseling session at the community pharmacy; patients in the control group had no session and only completed questionnaires. For the purpose of this study, only data from patients who had a counseling session were used.

At each participating pharmacy, one pharmacist and one pharmacy technician performed individual counseling sessions with patients with asthma and/or COPD. Each pharmacist selected a pharmacy technician from his/her own team, so 12 pharmacists and 12 technicians held sessions. Each patient had one session with either a pharmacist or a pharmacy technician. The number of counseling sessions depended on the number of participating patients in that particular pharmacy and on an agreed maximum number of participants per pharmacy.

To get an authentic impression of pharmacists' and technicians' communication styles, only general instructions were provided about the counseling sessions, that is, "discuss the patient's experience with ICS use, the effectiveness and (possible) side-effects of ICS, and whether the patient uses ICS in an appropriate way".

\section{Ethical approval}

The Medical Ethics Committee of the University Medical Center Utrecht assessed the research proposal and concluded that ethical approval was not required because the study did not fall within the remit of the Law on Medical Scientific Research involving Human Beings.

\section{Participants}

Patient recruitment took place through the participating pharmacies. Anatomical Therapeutic Chemical classification system codes for ICS and combination products of $\beta 2$-agonists and ICS were used to determine in the pharmacy system to which patients' ICS had been dispensed. Patients were eligible if they were aged 18 years or older, had been diagnosed with asthma or COPD (based on self-report), had been using ICS for at least 1 year, and had filled two or more ICS prescriptions in the preceding year. Patients living in an institution were excluded because they might receive other or additional care and counseling, which might influence the generalizability of the study results. Patients did not receive a reimbursement for participation.

\section{Procedure}

At each pharmacy, all eligible patients were invited to participate in the study, up to a maximum of 200 patients per pharmacy. If there were $>200$ eligible patients, a random subset of 200 patients were selected. An information pack was sent by post to 1,952 potentially eligible patients by their pharmacist, containing an invitation letter, a questionnaire, and an informed consent form. Patients were informed about the procedure and the videotaping of the consultations, and they had the opportunity to ask questions about the study and the videotaping of the session before consenting to participation. The completed questionnaire and a signed informed consent form had to be returned for study participation. A researcher contacted the participants by phone to make an appointment for a counseling session with a pharmacist or technician within 2 months after inclusion. The schedules for pharmacists and technicians had been determined beforehand Depending on patients' preferences for a date or time for an appointment, the consultation was scheduled. These sessions were performed in Dutch, took place in a private room, and were recorded by an unmanned camera, which was directed at the pharmacist or technician.

\section{Materials and measurements Questionnaire}

The questionnaire consisted of questions about sociodemographics, the medical indication for the ICS prescription (asthma/asthma symptoms and/or COPD or unknown), and other questions related to the patient's medical condition and ICS use.

\section{Communication measurements}

Analyses of the video-recorded sessions were performed using an extended version of the Roter interaction analysis system (RIAS). RIAS is a validated instrument for coding both provider and patient communication, applicable to the pharmacy setting. ${ }^{33-35}$ Each small meaningful unit of speech (an utterance) is coded.

RIAS distinguishes two categories of communication: 1) affective or socioemotional communication and 
2) instrumental communication, further divided into task- and process-oriented communication. Affective communication includes expressions of empathy, concern, optimism, and understanding, which contribute to a therapeutically effective provider-patient relationship. ${ }^{36}$ Instrumental communication focuses on the exchange of medical and psychosocial information and advice (task-oriented communication) and process-oriented utterances that guide the process of the conversation, like giving instructions (ie, "orientations").

The four main task-oriented RIAS categories are 1) medical, 2) therapeutic, 3) lifestyle/social, and 4) psychosocial. For the purpose of this study, the task-oriented categories were further divided into 36 content categories, including asthma- and COPD-specific items derived from existing communication literature and guidelines for asthma and COPD, ${ }^{31}$ from the consumer quality index asthma/ COPD,${ }^{30}$ and from the Dutch pharmacy COPD guideline. ${ }^{32}$ This literature was used to indicate topics that are relevant for patients with asthma and/or COPD and that could be discussed during counseling sessions. Examples of specific medical and therapeutic content categories are medical history, current health status, ICS side effects, and ICS adherence. Each main category furthermore had one "residual category" for utterances that belonged to that particular main category but did not fit into any of the specified categories (ie, "medical, other", "ICS, other", "lifestyle, other", and "psychosocial ICS, other"). Utterances about the study or comments about the recording were labeled as "other". For each task-oriented utterance, it was established whether it concerned a question ("question"), the provision of information ("giving information"), or an advice for the patient to change behavior ("counseling”) (Table S1).

Furthermore, the proportion of utterances made by the pharmacists, pharmacy technicians, and patients was determined as a global indicator of conversational contribution.

The videotapes were coded by three coders from the research team. Observer XT7 software was used to code the communication directly from the videotapes. ${ }^{37}$

\section{Interrater reliability}

Interrater reliability was tested using the intraclass correlation coefficient (ICC) and calculated for all main categories with a mean frequency $>2 \%$, except for the "other" categories. ${ }^{38}$ One coder recoded a random $10 \%$ of the two main coders' consultations. ICC reliability averaged 0.85 (range $0.37-0.98$ ) for pharmacist and technician categories and $0.89(0.70-0.97)$ for patient categories, which indicates moderate to good reliability, except for the category "orientations", which had an
ICC of 0.37 . Given the low ICC of the orientations category, no results are reported for this category.

\section{Statistical analysis}

Multilevel analysis was carried out to allow for the clustering of patients in pharmacies. Weighted mean and standard errors of all communication categories were calculated for pharmacists and technicians separately and have been reported. To control for visit length, weighted mean per category was divided by the total number of utterances and multiplied by $100 \%$.

Furthermore, we calculated the number of sessions in which a particular content category was mentioned by the pharmacist or the technician once only, more than once, or not at all, whether as a question, piece of advice, or information. Analyses were performed using MLwiN Version 2.25. To determine whether patients' sociodemographic characteristics differed between the pharmacists' sample and those of pharmacy technicians, two-sample proportion tests and Student's $t$-tests were performed using Stata Version 12.1. Descriptive statistics were calculated using Stata Version 12.1.

\section{Results \\ Study sample}

A total of 429 patients returned a completed consent form and questionnaire. One hundred and ninety-nine patients were assigned to the intervention group, of whom 30 patients (15.1\%) dropped out for one of the following reasons: they could not be reached by phone/email, involuntary withdrawal (eg, due to poor health), were no longer using ICS, had recently had a session about ICS or asthma, or did not want to participate after all. One hundred and sixty-nine patients received a counseling session at their pharmacy. Of these, 93 patients $(55.0 \%)$ were female and $76(45.0 \%)$ male. The mean age was 63.1 years $(\mathrm{SD}=13.9)$. Half of the 12 pharmacists were male and all the 12 technicians were female. The mean age of the pharmacists was 36.7 years $(\mathrm{SD}=11.5)$, and the mean age for technicians was 35.4 years $(\mathrm{SD}=11.9)$ (Table 1). The pharmacists performed 7.2 sessions on average (range: $3-11$ sessions) and pharmacy technicians 6.9 sessions (range: 3-10 sessions). The sociodemographic characteristics of patients in the pharmacists' sample did not differ significantly from those in the pharmacy technicians' sample.

\section{Counseling sessions}

Pharmacists' counseling sessions lasted significantly longer than those of pharmacy technicians (mean \pm standard error: $16.46 \pm 0.80$ minutes and $11.34 \pm 0.80$ minutes, respectively; 
Table I Patient, pharmacist, and pharmacy technician characteristics and summary of pharmacist-patient and technician-patient communication

\begin{tabular}{|c|c|c|c|}
\hline & $\begin{array}{l}\text { Pharmacist-patient } \\
\text { sessions } n=86\end{array}$ & $\begin{array}{l}\text { Technician-patient sessions } \\
n=83\end{array}$ & Total $n=169$ \\
\hline \multicolumn{4}{|l|}{ Patient characteristics } \\
\hline Female & 47 (54.7\%) & 46 (55.4\%) & $93(55.0 \%)$ \\
\hline \multicolumn{4}{|l|}{ Age, years } \\
\hline Mean (years) $\pm S D$ (range) & $63.7 \pm 13.4(26-88)$ & $62.5 \pm \mid 4.5(28-87)$ & $63.1 \pm 13.9(26-88)$ \\
\hline \multicolumn{4}{|l|}{ Age group, years } \\
\hline $18-44$ & $8(9.3 \%)$ & 12 (I4.5\%) & 20 (1 I.8\%) \\
\hline $45-64$ & $32(37.2 \%)$ & 29 (34.9\%) & 61 (36.1\%) \\
\hline$>64$ & $46(53.5 \%)$ & $42(50.6 \%)$ & $88(52.1 \%)$ \\
\hline \multicolumn{4}{|l|}{ Educational level ${ }^{\mathrm{a}, \mathrm{b}}$} \\
\hline Low & $33(38.4 \%)$ & 37 (44.6\%) & 70 (4I.4\%) \\
\hline Intermediate & 17 (19.8\%) & $16(19.3 \%)$ & $33(19.5 \%)$ \\
\hline High & 35 (40.7\%) & $29(34.9 \%)$ & $64(37.9 \%)$ \\
\hline \multicolumn{4}{|l|}{ Diagnosis ${ }^{c}$} \\
\hline Asthma & 34 (39.5\%) & 34 (4I.0\%) & $68(40.2 \%)$ \\
\hline COPD & 21 (24.4\%) & $21(25.3 \%)$ & $42(24.9 \%)$ \\
\hline Asthma and COPD & 17 (19.8\%) & $16(19.3 \%)$ & $33(19.5 \%)$ \\
\hline Unknown & $14(16.3 \%)$ & $12(14.5 \%)$ & $26(15.4 \%)$ \\
\hline
\end{tabular}

Pharmacist and pharmacy technician characteristics

Age group, years

$\begin{array}{lll}\text { Mean (years) } \pm S D \text { (range) } & 36.7 \pm 11.5(24-57) & 35.4 \pm 11.9(23-53) \\ \text { Female } & 6(50 \%) & 12(100 \%) \\ \begin{array}{l}\text { Number of years since graduating } \\ \text { Mean (years) } \pm S D \text { (range) }\end{array} & 11.1 \pm 10.4(0-32) & 14.4 \pm 11.8(2-34)\end{array}$

Pharmacist-patient and pharmacy technician-patient communication

\begin{tabular}{lllll}
\cline { 2 - 5 } & Pharmacist & Patient & Technician & Patient \\
\cline { 2 - 5 } Total communication $^{\text {d }}$ & $55.0 \%$ & $45.0 \%$ & $53.2 \%$ & $46.8 \%$ \\
$\quad$ Affective communication & $20.2 \%$ & $16.7 \%$ & $19.4 \%$ & $16.3 \%$ \\
$\quad \begin{array}{l}\text { Instrumental communication } \\
\text { Session length }\end{array}$ & $34.8 \%$ & $28.3 \%$ & $33.8 \%$ & $30.5 \%$ \\
$\quad$ Mean $^{\text {e }}$ (minutes) \pm SE (range) & $16.46 \pm 0.80(5.42-33.30)$ & & $11.34 \pm 0.80(4.23-26.73)$ & \\
\hline
\end{tabular}

Notes: ${ }^{2} T w o$ missing. 'Low: no education, primary school, prevocational secondary education (VMBO), lower vocational secondary education (LBO), junior general secondary education (MULO/MAVO); intermediate: upper vocational secondary education (MBO), senior general secondary education (HAVO), preuniversity education (VWO); high: university of applied sciences (HBO), university (WO). 'Diagnosis based on self-report. ${ }^{\mathrm{c}}$ Percentages are based on weighted mean. ${ }^{\mathrm{W}} \mathrm{Weighted} \mathrm{mean.}$

Abbreviations: COPD, chronic obstructive pulmonary disease; SE, standard error.

$P<0.001)$. Both categories of professionals talked more than patients in terms of the percentage of utterances (pharmacists accounted for $55.0 \%$ of the utterances in their sessions, technicians $53.2 \%$ ), and most of the communication consisted of instrumental talk (Table 1).

\section{Affective and process-oriented communication}

Table 2 shows the weighted mean of the affective and process-oriented communication behaviors per counseling session. Pharmacists and technicians mainly exhibited social behavior, expressed approval and agreement, and gave verbal attention and reassurance to patients. Patients showed similar behaviors and also expressed concerns or worries. With respect to process-oriented communication, pharmacists and technicians paraphrased and checked for understanding and asked the patient for an opinion at least once per session. Patients mainly paraphrased and checked for understanding.

\section{Task-oriented communication}

Several medical and therapeutic topics were discussed during the counseling sessions (Tables 3 and 4). In the following paragraphs, we describe the topics on which on average at least one utterance per session was made by pharmacists, technicians, or patients.

\section{Medical and therapeutic communication}

Pharmacists and technicians made at least one utterance per session about each of the following topics: "medical, other", 
Table 2 Weighted mean (SE) of affective behaviors and process-oriented behaviors of pharmacists, technicians, and patients in counseling sessions

\begin{tabular}{|c|c|c|c|c|}
\hline & \multicolumn{2}{|c|}{$\begin{array}{l}\text { Pharmacists' and technicians' } \\
\text { behavior }\end{array}$} & \multicolumn{2}{|l|}{ Patients' behavior } \\
\hline & Pharmacists & Technicians & Pharmacists' sessions & Technicians' sessions \\
\hline Affective part ${ }^{\mathrm{a}}$ & $89.02(5.48)^{*}$ & $63.72(5.53)^{*}$ & $73.55(5.06)^{* *}$ & $53.66(5.12)^{* *}$ \\
\hline Social behavior & $10.55(1.68)^{* *}$ & $4.86(1.70)^{* *}$ & $10.47(2.01)$ & $6.52(2.04)$ \\
\hline Approval & $6.91(0.56)^{*}$ & $3.68(0.56)^{*}$ & $1.70(0.24)$ & $\mathrm{I} .45(0.24)$ \\
\hline Agreements & $59.95(4.91)^{* *}$ & $48.50(4.94)^{* *}$ & $54.54(3.67)^{*}$ & $39.6 I(3.7 I)^{*}$ \\
\hline Verbal attention & $3.91(0.56)^{* *}$ & $2.00(0.57)^{* *}$ & $0.12(0.04)$ & $0.06(0.04)$ \\
\hline Shows concern or worry & $0.44(0.09)^{* * * *}$ & $0.15(0.10)^{* * *}$ & $1.95(0.37)$ & $1.37(0.38)$ \\
\hline Reassurance & $7.77(0.62)^{*}$ & $5.16(0.63)^{*}$ & $3.82(0.42)$ & $3.40(0.43)$ \\
\hline Disagree & $0.24(0.05)^{* *}$ & $0.05(0.05)^{* *}$ & $0.95(0.57)$ & $1.39(0.57)$ \\
\hline \multicolumn{5}{|l|}{ Instrumental part } \\
\hline Process-oriented ${ }^{\mathrm{a}}$ & $28.63(2.15)^{*}$ & $21.45(2.16)^{*}$ & $4.69(0.62)$ & $4.08(0.62)$ \\
\hline Orientations $^{\mathrm{b}}$ & $8.26(0.98)^{*}$ & $4.15(0.98)^{*}$ & $0.28(0.07)$ & $0.14(0.07)$ \\
\hline Paraphrase/check for understanding & $|7.9|(1.70)^{* * *}$ & $15.25(1.70)^{* * *}$ & $4.19(0.57)$ & $3.73(0.57)$ \\
\hline Bid for repetition & $0.04(0.02)$ & $0.05(0.02)$ & $0.14(0.05)$ & $0.14(0.05)$ \\
\hline Ask for understanding & $0.56(0.13)$ & $0.44(0.13)$ & $0.06(0.03)$ & $0.07(0.03)$ \\
\hline Ask for opinion & $1.89(0.26)$ & $1.58(0.26)$ & $0.00(0.01)$ & $0.01(0.01)$ \\
\hline $\begin{array}{l}\text { Request for services } \\
\text { (patient category) }\end{array}$ & - & - & $0.04(0.02)$ & $0.00(0.02)$ \\
\hline
\end{tabular}

Notes: ${ }^{a}$ Totals have been calculated using the model and can be different from the total of individual categories. ${ }^{\mathrm{b}}$ Due to a low intraclass correlation coefficient (ICC), no results have been reported in this article for this category. *Significant at $P<0.001$. **Significant at $P<0.01$. ***Significant at $P<0.05$.

Abbreviation: SE, standard error.

inhaler and inhalation technique, non-ICS treatment (other medication used by the patient), ICS medicinal effects, side effects, dosage and time of inhalation of ICS, current health status, adherence to ICS, reason for use, and "ICS, other". In contrast to technicians, pharmacists also asked at least once about patients' current health status and medical effects, and gave information about explanation about asthma/COPD more than once.

Patients made at least one utterance per session about the following issues: inhaler and inhalation technique, non-ICS medication, "medical, other", dosage and time of inhalation, current health status, medical history, side effects, contact with health care providers about asthma/COPD, "ICS, other", ICS medicinal effects, ICS adherence, lung function test, contact with other health care providers about ICS, and reason for use.

\section{Lifestyle and social context communication}

Pharmacists gave some social context information, but other lifestyle and social context topics were not mentioned more than once. Patients gave information about physical activity, social context, the (living) environment, and "lifestyle, other".

\section{Psychosocial/feelings communication}

Concerning psychosocial information, patients gave information about how they felt about non-ICS medication and
"ICS, other". Pharmacists and technicians addressed almost no psychosocial topics.

\section{Differences in communication between pharmacists and pharmacy technicians in their sessions}

Below, we describe the differences between pharmacists and pharmacy technicians in communication during the counseling sessions. Only significant differences $(P<0.05)$ are reported.

Pharmacists used more affective utterances per session on average than technicians. Patients did not differ in the number of affective utterances in their communication with pharmacists or technicians, except for giving agreements. Patients agreed more frequently in sessions with pharmacists than with technicians.

With respect to process-oriented communication, pharmacists and technicians differed in the number of utterances paraphrasing/checking for understanding. Patients had the same number of process-oriented utterances during sessions with pharmacists and technicians.

\section{Medical and therapeutic information}

The pharmacists were more likely to ask questions about the following topics than the technicians: patients' current health status, reason for ICS use, and other medication. Questions about side effects were asked more often by 
Table 3 Weighted mean (SE) of task-oriented behaviors of pharmacists and pharmacy technicians during counseling sessions

\begin{tabular}{|c|c|c|c|c|c|c|}
\hline \multirow[t]{2}{*}{ Task-oriented communication } & \multicolumn{2}{|l|}{ Question } & \multicolumn{2}{|c|}{ Giving information } & \multicolumn{2}{|l|}{ Counseling } \\
\hline & Pharmacists & Technicians & Pharmacists & Technicians & Pharmacists & Technicians \\
\hline Medical $^{\mathrm{a}}$ & $3.42(0.43)^{*}$ & $1.86(0.43)^{*}$ & $9.25(1.35)^{*}$ & $3.60(1.36)^{*}$ & $0.50(0.19)$ & $0.44(0.19)$ \\
\hline Prevalence & 0 & 0 & $0.06(0.04)$ & $0.00(0.04)$ & 0 & 0 \\
\hline Prognosis & 0 & 0 & $0.02(0.01)$ & $0.00(0.01)$ & 0 & 0 \\
\hline Exacerbations & $0.06(0.02)$ & $0.00(0.02)$ & $0.06(0.04)$ & $0.04(0.04)$ & 0 & 0 \\
\hline Current health status & $1.31(0.19)^{*}$ & $0.62(0.19)^{*}$ & $1.87(0.28)$ & $1.31(0.28)$ & $0.00(00.1)$ & $0.01(0.01)$ \\
\hline Medical history & $0.37(0.10)$ & $0.20(0.10)$ & $0.31(0.08)$ & $0.22(0.08)$ & 0 & 0 \\
\hline Heredity & $0.00(0.05)^{* *}$ & $0.09(0.05)^{* *}$ & $0.03(0.05)$ & $0.09(0.05)$ & 0 & 0 \\
\hline $\begin{array}{l}\text { Contact other health care } \\
\text { providers about asthma/COPD }\end{array}$ & $0.49(0.10)$ & $0.54(0.10)$ & $0.53(0.14)$ & $0.37(0.14)$ & $0.15(0.11)$ & $0.39(0.11)$ \\
\hline Lung function test & $0.32(0.10)$ & $0.08(0.10)$ & $0.96(0.32)^{* *}$ & $0.13(0.32)^{* *}$ & $0.19(0.08)$ & $0.00(0.08)$ \\
\hline Explanation about asthma/COPD & $0.01(0.01)$ & $0.00(0.01)$ & $2.65(0.62)^{*}$ & $0.06(0.63)^{*}$ & 0 & 0 \\
\hline Medical, other & $0.85(0.21)^{* * *}$ & $0.33(0.21)^{* * *}$ & $2.74(0.58)$ & $\mathrm{I} .47(0.59)$ & $0.15(0.04)$ & $0.05(0.05)$ \\
\hline Therapeutic: ICS treatment ${ }^{\mathrm{a}}$ & $10.97(0.87)$ & $10.57(0.87)$ & $46.53(3.17)^{*}$ & $33.42(3.20)^{*}$ & $11.25(1.45)$ & $11.12(1.46)$ \\
\hline Reason for use/need & $0.45(0.10)^{* *}$ & $0.20(0.10)^{* *}$ & $1.03(0.14)^{* * *}$ & $0.54(0.14)^{* * *}$ & $0.05(0.03)$ & $0.05(0.03)$ \\
\hline Dosage & $1.27(0.16)$ & $0.95(0.16)$ & $3.30(0.35)^{*}$ & $1.27(0.35)^{*}$ & $0.86(0.17)$ & $0.53(0.17)$ \\
\hline Adherence & $0.42(0.10)$ & $0.45(0.10)$ & $1.38(0.26)$ & $1.21(0.26)$ & $0.17(0.14)^{* * *}$ & $0.49(0.14)^{* * *}$ \\
\hline Medicinal effects & I.II (0.23) & $0.83(0.23)$ & $7.36(0.75)^{*}$ & $3.30(0.76)^{*}$ & $0.02(0.01)$ & $0.00(0.01)$ \\
\hline Side effects & $1.36(0.29)^{* *}$ & $1.90(0.29)^{* *}$ & $3.87(0.56)$ & $3.84(0.57)$ & $0.13(0.04)$ & $0.05(0.04)$ \\
\hline Inhaler/inhalation & $5.05(0.48)$ & $4.76(0.48)$ & $25.29(2.24)^{* * *}$ & $19.50(2.26)^{* * *}$ & $9.4 \mathrm{I}(\mathrm{I} .4 \mathrm{I})$ & $9.49(1.42)$ \\
\hline $\begin{array}{l}\text { Contact other health care } \\
\text { providers about ICS }\end{array}$ & $0.40(0.10)$ & $0.55(0.10)$ & $0.39(0.10)$ & $0.48(0.10)$ & $0.50(0.12)$ & $0.47(0.12)$ \\
\hline Self-management & $0.05(0.02)^{* * *}$ & $0.00(0.02)^{* * *}$ & $0.17(0.07)$ & $0.07(0.07)$ & $0.01(0.01)$ & $0.00(0.01)$ \\
\hline Facilitators & $0.07(0.06)$ & $0.14(0.06)$ & $0.88(0.20)$ & $0.84(0.20)$ & $0.01(0.01)$ & $0.00(0.01)$ \\
\hline Barriers & $0.06(0.03)$ & $0.04(0.03)$ & $0.52(0.12)$ & $0.36(0.12)$ & 0 & 0 \\
\hline ICS, other & $0.69(0.12)$ & $0.71(0.13)$ & $2.23(0.47)$ & $1.99(0.47)$ & $0.06(0.03)$ & $0.06(0.03)$ \\
\hline Therapeutic: non-ICS treatment ${ }^{\mathrm{a}}$ & $2.31(0.27)^{* * *}$ & $\mathrm{I} .6 \mathrm{I}(0.27)^{* * *}$ & $12.34(1.32)^{* *}$ & $7.46(1.34)^{* *}$ & $1.21(0.31)$ & $1.33(0.32)$ \\
\hline Lifestyle/social $^{2}$ & $0.74(0.18)^{* * *}$ & $0.40(0.18)^{* * *}$ & $3.31(0.56)^{* *}$ & $\mathrm{I} .43(0.57)^{* *}$ & $0.17(0.07)$ & $0.08(0.07)$ \\
\hline Smoking & $0.22(0.10)$ & $0.19(0.10)$ & $0.38(0.14)$ & $0.18(0.14)$ & $0.03(0.02)$ & $0.00(0.02)$ \\
\hline Exercise & $0.05(0.03)$ & $0.04(0.03)$ & $0.43(0.14)$ & $0.12(0.14)$ & $0.08(0.05)$ & $0.05(0.05)$ \\
\hline Weight & 0 & 0 & 0 & 0 & 0 & 0 \\
\hline Nutrition & $0.02(0.01)$ & $0.00(0.01)$ & $0.11(0.06)$ & $0.02(0.06)$ & $0.01(0.01)$ & $0.00(0.01)$ \\
\hline Stress & 0 & 0 & $0.00(0.01)$ & $0.02(0.01)$ & 0 & 0 \\
\hline Drugs & $0.02(0.02)$ & $0.00(0.02)$ & $0.05(0.03)$ & $0.00(0.03)$ & 0 & 0 \\
\hline Influenza vaccine & $0.02(0.01)$ & $0.00(0.01)$ & $0.00(0.03)$ & $0.04(0.03)$ & 0 & 0 \\
\hline Living environment & $0.15(0.07)$ & $0.14(0.07)$ & $0.89(0.27)$ & $0.4 I(0.27)$ & $0.01(0.01)$ & $0.00(0.01)$ \\
\hline Social context & $0.07(0.02)$ & $0.02(0.02)$ & $1.20(0.28)^{* * *}$ & $0.52(0.28)^{* * *}$ & $0.01(0.02)$ & $0.04(0.02)$ \\
\hline Lifestyle, other & $0.19(0.07)^{* * *}$ & $0.01(0.07)^{* * *}$ & $0.30(0.09)$ & $0.17(0.09)$ & $0.02(0.01)$ & $0.00(0.01)$ \\
\hline Psychosocial/feelings ${ }^{\mathrm{a}}$ & $0.32(0.08)$ & $0.25(0.08)$ & $1.18(0.26)$ & $1.09(0.26)$ & $0.03(0.02)$ & $0.00(0.02)$ \\
\hline ICS concerns & $0.01(0.01)$ & $0.00(0.01)$ & $0.14(0.04)^{* * *}$ & $0.0 \mathrm{I}(0.04)^{* * *}$ & 0 & 0 \\
\hline ICS necessities & 0 & 0 & $0.08(0.04)$ & $0.10(0.04)$ & 0 & 0 \\
\hline Psychosocial ICS, other & $0.30(0.07)$ & $0.20(0.07)$ & $0.66(0.15)$ & $0.59(0.15)$ & $0.03(0.02)$ & $0.00(0.02)$ \\
\hline Non-ICS & $0.01(0.03)$ & $0.05(0.03)$ & $0.29(0.13)$ & $0.39(0.13)$ & 0 & 0 \\
\hline Other & $0.4 \mathrm{I}(0.08)^{*}$ & $0.04(0.08)^{*}$ & $20.72(1.52)^{* *}$ & $15.09(1.54)^{* *}$ & $0.01(0.01)$ & $0.00(0.01)$ \\
\hline Total $^{\mathrm{a}}$ & $18.15(1.22)^{*}$ & $14.70(1.22)^{*}$ & $93.31(5.57)^{*}$ & $61.87(5.6 \mathrm{I})^{*}$ & $13.13(1.57)$ & $12.90(1.58)$ \\
\hline
\end{tabular}

Notes: ${ }^{T}$ Totals have been calculated using the model and can be different from the total of individual categories. *Significant at $P<0.001$. **Significant at $P<0.01$. $* * *$ Significant at $P<0.05$.

Abbreviations: SE, standard error; COPD, chronic obstructive pulmonary disease; ICS, inhaled corticosteroids.

technicians than by pharmacists. Patients were more likely to ask questions about dosage and time of inhalation of ICS during sessions with pharmacists than with technicians. Pharmacists were more likely to give information about a lung function test, asthma/COPD, reason for ICS use, dosage and time of inhalation, ICS effects, inhaler and inhalation technique, and other medication. Patients were more likely to provide information to pharmacists than technicians about the following topics: medical history, dosage and time of inhalation, and barriers. Technicians were more likely to give advice about ICS adherence than pharmacists. 
Table 4 Weighted mean (SE) of task-oriented behaviors of patients in sessions with pharmacists and pharmacy technicians

\begin{tabular}{|c|c|c|c|c|}
\hline \multirow[t]{2}{*}{ Task-oriented communication } & \multicolumn{2}{|l|}{ Question } & \multicolumn{2}{|l|}{ Giving information } \\
\hline & Pharmacists' sessions & Technicians' sessions & Pharmacists' sessions & Technicians' sessions \\
\hline Medical $^{\mathrm{a}}$ & $0.33(0.07)^{*}$ & $0.12(0.07)^{*}$ & $22.94(2.20)^{*}$ & $|7.0|(2.24)^{*}$ \\
\hline Prevalence & $0.01(0.01)$ & $0.00(0.01)$ & 0 & 0 \\
\hline Prognosis & 0 & 0 & 0 & 0 \\
\hline Exacerbations & 0 & 0 & $0.21(0.10)$ & $0.15(0.10)$ \\
\hline Current health status & $0.06(0.03)$ & $0.06(0.03)$ & $5.52(0.53)$ & $4.31(0.53)$ \\
\hline Medical history & $0.03(0.02)$ & $0.01(0.02)$ & $5.01(0.66)^{* *}$ & $2.24(0.68)^{* *}$ \\
\hline Heredity & 0 & 0 & $0.11(0.12)$ & $0.30(0.12)$ \\
\hline $\begin{array}{l}\text { Contact other health care providers } \\
\text { about asthma/COPD }\end{array}$ & $0.02(0.01)$ & $0.01(0.01)$ & $2.83(0.4 I)$ & $2.46(0.42)$ \\
\hline Lung function test & $0.08(0.06)$ & $0.02(0.06)$ & $1.86(0.35)$ & $0.99(0.36)$ \\
\hline Explanation about asthma/COPD & $0.07(0.03)$ & $0.00(0.03)$ & $0.15(0.05)$ & $0.06(0.05)$ \\
\hline Medical, other & $0.06(0.02)$ & $0.02(0.02)$ & $7.33(1.47)$ & $6.62(1.49)$ \\
\hline Therapeutic: ICS treatment ${ }^{\mathrm{a}}$ & $2.62(0.35)$ & $2.28(0.36)$ & $44.19(3.08)$ & $40.72(3.10)$ \\
\hline Reason for use/need & $0.06(0.05)$ & $0.07(0.05)$ & $1.19(0.18)$ & $1.00(0.18)$ \\
\hline Dosage & $0.36(0.10)^{*}$ & $0.08(0.10)^{*}$ & $5.67(0.49)^{* * *}$ & $3.92(0.49)^{* * *}$ \\
\hline Adherence & $0.04(0.02)$ & $0.01(0.02)$ & $1.95(0.31)$ & $\mathrm{I} .43(0.3 \mathrm{I})$ \\
\hline Medicinal effects & $0.15(0.04)$ & $0.05(0.04)$ & $2.44(0.38)$ & $\mathrm{I} .82(0.39)$ \\
\hline Side-effects & $0.24(0.08)$ & $0.23(0.08)$ & $3.86(0.57)$ & $3.91(0.58)$ \\
\hline Inhaler/inhalation & $1.52(0.26)$ & $\mathrm{I} .64(0.26)$ & $22.76(2.11)$ & $22.64(2.12)$ \\
\hline $\begin{array}{l}\text { Contact other health care providers } \\
\text { about ICS }\end{array}$ & $0.08(0.02)$ & $0.02(0.02)$ & $1.62(0.30)$ & $2.11(0.30)$ \\
\hline Self-management & 0 & 0 & $0.16(0.09)$ & $0.29(0.09)$ \\
\hline Facilitators & $0.01(0.01)$ & $0.01(0.01)$ & $0.51(0.15)$ & $0.56(0.15)$ \\
\hline Barriers & $0.02(0.02)$ & $0.02(0.02)$ & $0.92(0.19)^{*}$ & $0.34(0.19)^{*}$ \\
\hline ICS, other & $0.14(0.04)$ & $0.13(0.04)$ & $2.81(0.29)$ & $2.47(0.29)$ \\
\hline Therapeutic: Non-ICS treatment ${ }^{\mathrm{a}}$ & $0.74(0.15)$ & $0.59(0.15)$ & I5.2I (I.49) & $12.40(1.52)$ \\
\hline Lifestyle/social $^{\mathrm{a}}$ & $0.17(0.05)^{* *}$ & $0.00(0.05)^{* *}$ & $9.29(1.43)^{*}$ & $5.74(1.45)^{*}$ \\
\hline Smoking & 0 & 0 & $0.99(0.3 \mathrm{I})$ & $0.62(0.3 \mathrm{I})$ \\
\hline Exercise & $0.05(0.02)$ & $0.00(0.02)$ & $1.99(0.34)^{*}$ & I.0I $(0.35)^{*}$ \\
\hline Weight & 0 & 0 & $0.00(0.09)$ & $0.18(0.09)$ \\
\hline Nutrition & 0 & 0 & $0.30(0.19)$ & $0.33(0.19)$ \\
\hline Stress & 0 & 0 & $0.01(0.03)$ & $0.04(0.03)$ \\
\hline Drugs & 0 & 0 & $0.16(0.08)$ & $0.00(0.08)$ \\
\hline Influenza vaccine & 0 & 0 & $0.04(0.06)$ & $0.18(0.06)$ \\
\hline (Living) environment & $0.07(0.04)$ & $0.00(0.04)$ & $1.52(0.25)^{* *}$ & $0.70(0.25)^{* *}$ \\
\hline Social context & $0.05(0.02)^{*}$ & $0.00(0.02)^{*}$ & $2.75(0.67)$ & $1.60(0.68)$ \\
\hline Lifestyle, other & $0.01(0.01)$ & $0.00(0.01)$ & $\mathrm{I} .43(0.37)$ & $0.96(0.37)$ \\
\hline Psychosocial/feelings $^{\mathrm{a}}$ & $0.09(0.04)$ & $0.05(0.04)$ & $5.84(0.78)$ & $5.37(0.79)$ \\
\hline ICS concerns & $0.05(0.02)$ & $0.02(0.02)$ & $0.98(0.21)^{*}$ & $0.45(0.2 \mathrm{I})^{*}$ \\
\hline ICS necessities & $0.02(0.01)$ & $0.00(0.01)$ & $0.51(0.13)$ & $0.44(0.13)$ \\
\hline Psychosocial ICS, other & $0.02(0.01)$ & $0.01(0.01)$ & $2.14(0.37)$ & $2.05(0.37)$ \\
\hline Non-ICS & $0.00(0.01)$ & $0.01(0.01)$ & $2.15(0.55)$ & $2.39(0.56)$ \\
\hline Other & $0.44(0.09)$ & $0.21(0.09)$ & $17.92(1.31)^{* *}$ & II.89 (I.34)** \\
\hline Total $^{\mathrm{a}}$ & $4.38(0.49)$ & $3.22(0.50)$ & II $5.40(6.28)^{* * *}$ & $92.77(6.36)^{* * *}$ \\
\hline
\end{tabular}

Notes: ${ }^{\top}$ Totals have been calculated in the model and can be different from the total of individual categories. ${ }^{*}$ Significant at $P<0.05$. ${ }^{*}$ Significant at $P<0.01$. $* * *$ Significant at $P<0.001$.

Abbreviations: SE, standard error; COPD, chronic obstructive pulmonary disease; ICS, inhaled corticosteroids.

\section{Lifestyle and social context information}

Pharmacists gave information about the social context more often than technicians. Patients gave information to pharmacists more often than to technicians about physical activity and the (living) environment.

\section{Psychosocial information/feelings}

Pharmacists and technicians differed in giving information regarding concerns about ICS, which was mentioned on average less than once a session. Patients also differed in giving information about this topic; they talked more 
often about ICS concerns in sessions with pharmacists than in sessions with technicians.

\section{Number of sessions in which topics were discussed}

Several clinically relevant topics were not discussed during many of the counseling sessions (Table S2). The comparison between pharmacists and technicians revealed that questions about current health status were asked more often by pharmacists than by technicians, in 26 versus eleven sessions, respectively. Pharmacists gave an explanation about asthma more than once in 22 sessions; technicians did so in three sessions. Pharmacists paid attention more than once to the reason for ICS use in 26 sessions and to dosage in 55 sessions, whereas technicians only did so in 12 and 25 sessions, respectively. Technicians asked more than once about side effects in 42 sessions, whereas pharmacists did so in 29 sessions. Lifestyle/social categories were hardly mentioned. Smoking, the (living) environment, and the social context were discussed in a couple of sessions. In addition, psychosocial aspects or feelings were seldom discussed.

\section{Outcomes controlled for visit length}

As stated before, pharmacists' sessions lasted longer than those of technicians. After controlling for visit length, most of the differences between pharmacists and technicians in communication behaviors remained significant. This was also the case for the differences found in patient communication during sessions with pharmacists and technicians after controlling for visit length (Table S3).

After controlling for visit length, differences between pharmacists' and technicians' communication remained significant for the following affective categories: social behavior, approval, verbal attention, showing concerns/worry, and disagreeing. The differences in the number of questions about current health status, heredity, "medical, other", side effects, "lifestyle, other", and "other" also remained significant. In addition, differences in paraphrasing/checking for understanding and giving information about the lung function test, explanation about asthma/COPD, dosage, medicinal effects, and ICS concerns were still significant after controlling for visit length, as well as counseling about adherence.

For patient utterances, the number of questions about dosage, giving information about medical history, the (living) environment, and ICS concerns still differed between sessions with pharmacists and technicians after controlling for visit length.

\section{Discussion}

Many important issues were addressed during the counseling sessions. However, pharmacists and technicians could pay more attention to some topics. Some differences in communication have been found between pharmacists and pharmacy technicians.

The counseling sessions consisted largely of instrumental talk. Medical and therapeutic topics were frequently discussed, such as current health status, inhaler and inhalation technique, side effects, dosage and time of inhalation, and ICS medicinal effects. Pharmacists and pharmacy technicians discussed adherence to a limited degree only. In this respect, counseling sessions reflect those of other health care providers: this topic is often avoided during patient-provider interactions. ${ }^{21,31}$

Lifestyle and psychosocial issues were hardly addressed by pharmacists and technicians, while patients did mention these topics. As concerns have relevance for adherence to medication, ${ }^{18,39}$ there might be room for improvement in discussing patients' concerns about ICS and why it is necessary to use them. Pharmacists and technicians could also pay more attention to lifestyle topics, such as smoking habits and exercise.

Pharmacists and technicians talked with the patients extensively about the inhaler and inhalation technique, clearly the main aim of the session. In addition, medication other than ICS (the category "non-ICS treatment") was often discussed, such as bronchodilators (relievers). Because bronchodilators are often used in combination with ICS, pharmacists and technicians probably discuss these to check the inhalation technique as well as the effectiveness of the medication.

Remarkably, patients raised very few questions. On average, they only asked questions about the inhaler and inhalation technique more than once per session. This suggests either that they did not have much to ask or that there was not enough opportunity to pose questions. Pharmacists and technicians could invite patients more explicitly to ask questions.

An important aspect concerning pharmacist-patient communication is the way patients view the pharmacists' role (ie, tasks and responsibilities). A few studies have shown that some groups of patients have positive views about pharmacists' enhanced role in disease management and medication advice. ${ }^{40,41}$ However, other studies have found that patients do not perceive pharmacists as having a significant role in patient counseling, medication management, or monitoring; patients believe this to be primarily the task of physicians. ${ }^{42-44}$ These findings could be a reason for patients' 
possible reticence in posing questions and discussing medical, lifestyle, and psychosocial issues.

When comparing pharmacists' and technicians' sessions, pharmacists showed more affective behavior than technicians, and they also discussed some task-oriented topics more extensively. Although pharmacists' sessions lasted 1.5 times longer than those of technicians, these differences could not be fully explained by the longer visit length of the pharmacists' sessions. In contrast to pharmacists and technicians, patients did not differ in their affective and process-oriented communications when being counseled by one or the other professional.

Pharmacist and pharmacy technicians spoke more than patients (55.0\% and $53.2 \%$ of the utterances, respectively). Although this indicates that the providers made a greater conversational contribution than patients, we think the share of provider-patient communication is fairly balanced because the percentages are close to the middle (ie, $50 \%$ ).

We can compare our findings with only a few studies. Because of dissimilarities in study setting and health care providers, these findings are difficult to compare. When comparing our findings with general practitioner-patient communication, no remarkable differences were found. Pharmacists and technicians in our study showed $\sim 5 \%$ more affective behavior than general practitioners, which could be explained to a large extent by the many agreements that pharmacists and technicians expressed. ${ }^{20}$ General practitioners did not discuss lifestyle/social and psychosocial topics in much depth; however, pharmacists and technicians discussed them even less. Our findings also confirm the results of a simulated patient study of Chong et $\mathrm{al}^{25}$ about antidepressants, which showed that pharmacists' communication is merely focused on biomedical topics and that lifestyle and psychosocial topics are underexposed.

To our knowledge, this is the first study about communication during counseling sessions about ICS in the pharmacy. For future research, it would be interesting to study the relationship between the communication process and content on the one hand and outcomes on the other hand, such as patients' medication adherence, satisfaction, and symptom control. In addition, the experiences of patients and health care providers with the communication could be taken into account, to get insight into which communication styles enhance medication optimization, according to them.

\section{Strengths and limitations}

This study has several strengths. First, exploring the content of counseling sessions at the pharmacy has never been done before in such an extensive way, resulting in rich information about these sessions and an objective and quantitative presentation of the content of the verbal communication. In addition, this study did not only take pharmacists' communication into account but also looked at technicians' communication and compared the content of the sessions of these two categories of professionals.

However, there are some limitations. Selection bias could have occurred during pharmacy and patient recruitment. Participating pharmacies could be more engaged in patient counseling than the nonparticipating pharmacies, and participating patients might be more willing to communicate about ICS use, related problems, and feelings. In the Netherlands, counseling sessions are not yet routine activities in pharmacies, ${ }^{23}$ which implies that, in general, pharmacists and technicians are not very experienced in conducting counseling sessions about ICS. Therefore, the participating pharmacists and technicians might have better counseling skills than their nonparticipating colleagues. Hence, the generalizability of the findings is limited.

In addition, the pharmacists sent the invitation letter to patients. This might have resulted in bias as patients might feel obligated to participate in the study. However, it was stated clearly in the letter that participation was voluntary.

Finally, because counseling sessions are not performed very frequently, we provided general instructions to the pharmacists and technicians about which themes could be discussed during the sessions. Although these instructions were very broad, they might have influenced our results.

\section{Conclusion}

Both pharmacists and pharmacy technicians addressed many important medical and therapeutic topics in counseling sessions with patients with asthma and/or COPD, but they could pay more attention to ICS adherence and to lifestyle and psychosocial topics. The two categories of professionals differed in their communication: pharmacists exhibited more affective behavior than technicians and also discussed medical and therapeutic topics in particular more extensively.

\section{Practice implications}

Educational courses for pharmacists and pharmacy technicians could draw attention to the discussion of ICS adherence, lifestyle, and psychosocial topics in patient communication. Pharmacy technicians in particular could be encouraged to attend to other factors than purely the technical aspects of using ICS. 


\section{Acknowledgments}

We would like to thank Peter Spreeuwenberg for his statistical advice and Jorien Bijleveld for coding of the video recordings. We also thank all patients, pharmacists, and pharmacy technicians for their participation. We also thank the Royal Dutch Association for the Advancement of Pharmacy (KNMP) and the Dutch Association for Innovative Medicine (Nefarma) for their financial contributions to this study.

\section{Disclosure}

The authors report no conflicts of interest in this work.

\section{References}

1. Allemann SS, van Mil JW, Botermann L, Berger K, Griese N, Hersberger KE. Pharmaceutical care: the PCNE definition 2013. Int $J$ Clin Pharm. 2014;36(3):544-555.

2. Berenguer B, La CC, de la Matta MJ, Martin-Calero MJ. Pharmaceutical care: past, present and future. Curr Pharm Des. 2004;10(31):3931-3946.

3. Mehuys E, Van Bortel L, De Bolle L, et al. Effectiveness of a community pharmacist intervention in diabetes care: a randomized controlled trial J Clin Pharm Ther. 2011;36(5):602-613.

4. Stuurman-Bieze AG, Hiddink EG, van Boven JF, Vegter S. Proactive pharmaceutical care interventions decrease patients' nonadherence to osteoporosis medication. Osteoporos Int. 2014;25(6):1807-1812.

5. Vrijens B, Belmans A, Matthys K, de Klerk E, Lesaffre E. Effect of intervention through a pharmaceutical care program on patient adherence with prescribed once-daily atorvastatin. Pharmacoepidemiol Drug Saf. 2006;15(2):115-121.

6. Sabate E. Adherence to Long-Term Therapies: Evidence for Action. Geneva: WHO; 2003. ISBN 9241545992.

7. Sin DD, McAlister FA, Man SF, Anthonisen NR. Contemporary management of chronic obstructive pulmonary disease: scientific review. J Amer Med Assoc. 2003;290(17):2301-2312.

8. Spencer S, Calverley PM, Burge PS, Jones PW. Impact of preventing exacerbations on deterioration of health status in COPD. Eur Respir J. 2004;23(5):698-702.

9. Sims EJ, Price D, Haughney J, Ryan D, Thomas M. Current control and future risk in asthma management. Allergy Asthma Immunol Res. 2011 3(4):217-225.

10. Sullivan SD, Wenzel SE, Bresnahan BW, et al. Association of control and risk of severe asthma-related events in severe or difficult-to-treat asthma patients. Allergy. 2007;62(6):655-660.

11. Hermosa JL, Sánchez CB, Rubio MC, Mínguez MM, Walther JL. Factors associated with the control of severe asthma. J Asthma. 2010;47(2): 124-130.

12. Jobin MS, Moisan J, Bolduc Y, Dorval E, Boulet LP, Grégoire JP. Factors associated with the appropriate use of asthma drugs. Can Respir J. 2011;18(2):97-104.

13. Saini B, LeMay K, Emmerton L, et al. Asthma disease managementAustralian pharmacists' interventions improve patients' asthma knowledge and this is sustained. Patient Educ Couns. 2011;83(3):295-302.

14. Baan D, Heijmans M, Spreeuwenberg P, Rijken M. Zelfmanagement vanuit het perspectief van mensen met astma of COPD [Selfmanagement from the Perspective of Persons with Asthma or COPD]. Utrecht: NIVEL; 2012. Available from: http://www.longalliantie.nl/publicaties. Accessed February 2, 2012. Dutch.

15. LAN [Lung Alliance the Netherlands]. Goed gebruik inhalatiemedicatie astma en COPD [Proper Use Inhalation Medication Asthma and COPD]. 2011. Dutch.

16. Giraud V, Allaert FA, Roche N. Inhaler technique and asthma: feasability and acceptability of training by pharmacists. Respir Med. 2011; 105(12):1815-1822.
17. Horne R. Compliance, adherence, and concordance: implications for asthma treatment. Chest. 2006;130(1 suppl):65S-72S.

18. Menckeberg TT, Bouvy ML, Bracke M, et al. Beliefs about medicines predict refill adherence to inhaled corticosteroids. J Psychosom Res. 2008;64(1):47-54.

19. Van Steenis M, Driesenaar J, Bensing J, et al. Relationship between medication beliefs, self-reported and refill adherence, and symptoms in patients with asthma using inhaled corticosteroids. Patient Prefer Adherence. 2014;8:83-91.

20. Van den Brink-Muinen A, Van Dulmen AM, Schellevis FG, Bensing JM. Tweede Nationale Studie naar ziekten en verrichtingen in de huisartspraktijk. Oog voor communicatie: huisarts-patient communicatie in Nederland [Second Dutch National Study on Diseases and Performances in General Practice]. Utrecht: NIVEL; 2004.

21. Van Dulmen S, Van Bijnen E. What makes them (not) talk about proper medication use with their patients? An analysis of the determinants of GP communication using reflective practice. Int J Person Centered Med. 2011;1:27-34

22. Zolnierek KB, Dimatteo MR. Physician communication and patient adherence to treatment: a meta-analysis. Med Care. 2009;47(8): 826-834.

23. Pronk M, Blom L, Jonkers R, Bakker A. Community pharmacy and patient-oriented activities: the Dutch case. Patient Educ Couns. 2002; 46(1):39-45.

24. Shah B, Chewning B. Conceptualizing and measuring pharmacistpatient communication: a review of published studies. Res Social Adm Pharm. 2006;2(2):153-185.

25. Chong WW, Aslani P, Chen TF. Pharmacist-patient communication on use of antidepressants: a simulated patient study in community pharmacy. Res Social Adm Pharm. 2014;10(2):419-437.

26. Murad MS, Chatterley T, Guirguis LM. A meta-narrative review of recorded patient-pharmacist interactions: exploring biomedical or patient-centered communication? Res Social Adm Pharm. 2014;10(1): $1-20$.

27. van Mil JW. Pharmaceutical care in community pharmacy: practice and research in the Netherlands. Ann Pharmacother. 2005;39(10): $1720-1725$.

28. Blom AThG. Developing Patient Education in Community Pharmacy [dissertation]. Utrecht: Universiteit Utrecht; 1996.

29. Henselmans I, Heijmans M, Rademakers J, van Dulmen S. Participation of chronic patients in medical consultations: patients' perceived efficacy, barriers and interest in support. Health Expect. 2015;18(6): 2375-2388.

30. Jansen DL, Bloemendal E, Rademakers J. CQ-Index astma en COPD: Meetinstrumentontwikkeling en onderzoek naar het discriminerend vermogen. Kwaliteit van van zorg bij astma en COPD vanuit het perspectief van patiënten met astma en COPD [Consumer Quality Index Asthma and COPD]. Utrecht: NIVEL; 2010. Dutch.

31. Prins M, Schoen T, Doggen C, Van Dijk L, van Dulmen S. Het betrekken van het perspectief van patiënt en voorschrijver bij het verbeteren van de communicatie over geneesmiddelen en therapietrouw [Involving Patient's and Prescriber's Perspective in Improving Communication about Medicines and Adherence]. Utrecht: NIVEL; 2008. Dutch.

32. KNMP [Royal Dutch Association for the Advancement of Pharmacy]. Richtlijn COPD [Guideline COPD]. 2011. Utrecht. Dutch. Available from: http://www.knmp.nl/patientenzorg/aandoening-en-preventie/ astma-copd/knmp-richtlijn-copd. Accessed March 1, 2012.

33. Roter $\mathrm{D}$, Larson $\mathrm{S}$. The Roter interaction analysis system (RIAS): utility and flexibility for analysis of medical interactions. Patient Educ Couns. 2002;46(4):243-251.

34. Cavaco A, Roter D. Pharmaceutical consultations in community pharmacies: utility of the Roter Interaction Analysis System to study pharmacist-patient communication. Int J Pharm Pract. 2010;18(3): 141-148.

35. Roter D. The Roter Interaction Analysis System (RIAS) Coding Manual. John Hopkins University, Baltimore: 1991. 
36. Bensing JM, Verhaak PFM. Communication in medical encounters: towards a health psychology perspective. In: Kaptein AA, Weinman J, editors. Health Psychology, an Introduction. Oxford: Blackwell; 2004: 261-288.

37. Noldus L, Trienes R, Hendriksen A, Jansen H, Jansen R. The observer video-pro: new software for the collection, management and preservation of time-structured data from videotapes and digital media-files. Behav Res Methods Instrum Comput. 2000;32:197-206.

38. Ford S, Fallowfield L, Lewis S. Doctor-patient interactions in oncology. Soc Sci Med. 1996;42(11):1511-1519.

39. Horne R, Weinman J. Patients' beliefs about prescribed medicines and their role in adherence to treatment in chronic physical illness. J Psychosom Res. 1999;47(6):555-567.

40. Naik-Panvelkar P, Saini B, LeMay KS, et al. A pharmacy asthma service achieves a change in patient responses from increased awareness to taking responsibility for their asthma. Int J Pharm Pract. 2015;23(3): $182-191$.
41. Twigg MJ, Poland F, Bhattacharya D, Desborough JA, Wright DJ. The current and future roles of community pharmacists: views and experiences of patients with type 2 diabetes. Res Social Adm Pharm. 2013;9(6): 777-789.

42. Schommer JC, Gaither CA. A segmentation analysis for pharmacists' and patients' views of pharmacists' roles. Res Social Adm Pharm. 2014; 10(3):508-528.

43. Worley MM, Schommer JC, Brown LM, et al. Pharmacists' and patients' roles in the pharmacist-patient relationship: are pharmacists and patients reading from the same relationship script? Res Social Adm Pharm. 2007;3(1):47-69.

44. Law AV, Ray MD, Knapp KK, Balesh JK. Unmet needs in the medication use process: perceptions of physicians, pharmacists, and patients. J Am Pharm Assoc. 2003;43(3):394-402. 


\section{Supplementary materials}

Table SI Examples of statements for RIAS categories

\begin{tabular}{|c|c|}
\hline RIAS category & Examples of statements \\
\hline \multicolumn{2}{|l|}{ Affective part } \\
\hline Social behavior & At the beginning of the session: Pharmacist: "How are you?" \\
\hline Approval & (Patient shows inhaler technique) Pharmacist: "That looks very good!" \\
\hline Agreements & "Hmm, hmm" “Okay" \\
\hline Verbal attention & Pharmacist: "I understand how you must be feeling" \\
\hline Shows concern or worry & Patient: "I hope it's nothing serious" \\
\hline (Ask for) reassurance & Patient: "My asthma is much better" \\
\hline Disagree & Patient: “That's impossible!” \\
\hline \multicolumn{2}{|l|}{ Instrumental part } \\
\hline \multicolumn{2}{|l|}{ Process-oriented } \\
\hline Orientations & Pharmacist: "Can you show me how you use your inhaler, please?" \\
\hline \multirow[t]{2}{*}{ Paraphrase/check for understanding } & (Pharmacist: "You have to hold your breath for ten seconds") \\
\hline & Patient: "Ten seconds?" \\
\hline Bid for repetition & Patient: "What did you say?" \\
\hline Ask for understanding & Pharmacist: "Do you follow?" \\
\hline Ask for opinion & Pharmacist: "Any questions?" \\
\hline Request for services (patient category) & Patient: "Could you contact the doctor for this, please?" \\
\hline \multicolumn{2}{|l|}{ Task-oriented communication } \\
\hline Medical & Pharmacist: "Do you have asthma?" \\
\hline Therapeutic & Patient: "I take two puffs per day" \\
\hline Lifestyle/social & Pharmacist: "Do you smoke?" \\
\hline Psychosocial/feelings & Patient: "I'm worried about the long-term effects of ICS" \\
\hline Other & Patient: "Where can I fill in the questionnaire?" \\
\hline
\end{tabular}

Abbreviations: RIAS, Roter interaction analysis system; ICS, inhaled corticosteroids. 
Table S2 The number of sessions in which a topic is discussed zero times, once, or more than once (divided into questions, giving information, and counseling)

\begin{tabular}{|c|c|c|c|c|c|c|c|}
\hline \multicolumn{2}{|c|}{ Question } & \multicolumn{3}{|c|}{ Giving information } & \multicolumn{3}{|c|}{ Counseling } \\
\hline 0 & $>1$ & 0 & I & $>1$ & 0 & I & $>1$ \\
\hline
\end{tabular}

\begin{tabular}{|c|c|c|c|c|c|c|c|c|c|}
\hline \multicolumn{10}{|l|}{ Medical } \\
\hline \multicolumn{10}{|l|}{ Prevalence } \\
\hline Pharmacists & 86 & 0 & 0 & 85 & 0 & 1 & 86 & 0 & 0 \\
\hline Technicians & 83 & 0 & 0 & 83 & 0 & 0 & 83 & 0 & 0 \\
\hline \multicolumn{10}{|l|}{ Prognosis } \\
\hline Pharmacists & 86 & 0 & 0 & 84 & 2 & 0 & 86 & 0 & 0 \\
\hline Technicians & 83 & 0 & 0 & 83 & 0 & 0 & 83 & 0 & 0 \\
\hline \multicolumn{10}{|l|}{ Exacerbations } \\
\hline Pharmacists & 82 & 3 & 1 & 83 & 2 & 1 & 86 & 0 & 0 \\
\hline Technicians & 83 & 0 & 0 & 82 & 0 & 1 & 83 & 0 & 0 \\
\hline \multicolumn{10}{|c|}{ Current health status } \\
\hline Pharmacists & 40 & 17 & 29 & 35 & 22 & 29 & 86 & 0 & 0 \\
\hline Technicians & 49 & 23 & 11 & 41 & 20 & 22 & 82 & 1 & 0 \\
\hline \multicolumn{10}{|l|}{ Medical history } \\
\hline Pharmacists & 65 & 13 & 8 & 69 & 10 & 7 & 86 & 0 & 0 \\
\hline Technicians & 69 & 10 & 4 & 72 & 8 & 3 & 83 & 0 & 0 \\
\hline \multicolumn{10}{|l|}{ Heredity } \\
\hline Pharmacists & 86 & 0 & 0 & 84 & 1 & 1 & 86 & 0 & 0 \\
\hline Technicians & 77 & 4 & 2 & 79 & 2 & 2 & 83 & 0 & 0 \\
\hline \multicolumn{10}{|c|}{ Contact other health care providers about asthma/COPD } \\
\hline Pharmacists & 60 & 15 & 11 & 64 & 12 & 10 & 78 & 4 & 4 \\
\hline Technicians & 56 & 16 & 11 & 69 & 7 & 7 & 70 & 7 & 6 \\
\hline \multicolumn{10}{|c|}{ Lung function test } \\
\hline Pharmacists & 72 & 8 & 6 & 62 & 10 & 14 & 81 & I & 4 \\
\hline Technicians & 78 & 4 & I & 74 & 5 & 4 & 83 & 0 & 0 \\
\hline \multicolumn{10}{|c|}{ Explanation about asthma/COPD } \\
\hline Pharmacists & 85 & 1 & 0 & 59 & 5 & 22 & 86 & 0 & 0 \\
\hline Technicians & 83 & 0 & 0 & 79 & I & 3 & 83 & 0 & 0 \\
\hline \multicolumn{10}{|l|}{ Medical, other } \\
\hline Pharmacists & 56 & 10 & 20 & 45 & 10 & 31 & 77 & 6 & 3 \\
\hline Technicians & 69 & 6 & 8 & 55 & 9 & 19 & 80 & 2 & I \\
\hline \multicolumn{10}{|c|}{ Therapeutic: ICS treatment } \\
\hline \multicolumn{10}{|c|}{ Reason for use/need } \\
\hline Pharmacists & 54 & 25 & 7 & 45 & 15 & 26 & 82 & 4 & 0 \\
\hline Technicians & 64 & 18 & I & 59 & 12 & 12 & 81 & 1 & I \\
\hline \multicolumn{10}{|c|}{ Dosage/inhalation } \\
\hline Pharmacists & 31 & 22 & 33 & 18 & 13 & 55 & 59 & 11 & 16 \\
\hline Technicians & 32 & 29 & 22 & 36 & 22 & 25 & 62 & 13 & 8 \\
\hline \multicolumn{10}{|l|}{ Adherence } \\
\hline Pharmacists & 60 & 19 & 7 & 45 & 16 & 25 & 76 & 8 & 2 \\
\hline Technicians & 55 & 21 & 7 & 49 & 12 & 22 & 67 & 8 & 8 \\
\hline \multicolumn{10}{|l|}{ Medicinal effects } \\
\hline Pharmacists & 40 & 25 & 21 & 10 & 8 & 68 & 84 & 2 & 0 \\
\hline Technicians & 43 & 22 & 18 & 13 & 16 & 54 & 83 & 0 & 0 \\
\hline Side effects & & & & & & & & & \\
\hline Pharmacists & 30 & 27 & 29 & 26 & 10 & 50 & 78 & 5 & 3 \\
\hline Technicians & 16 & 25 & 42 & 18 & 9 & 56 & 81 & 1 & 1 \\
\hline Inhaler/inhalatic & & & & & & & & & \\
\hline Pharmacists & 5 & 8 & 73 & 2 & 3 & 81 & 13 & 4 & 69 \\
\hline Technicians & 5 & 4 & 74 & 0 & 1 & 82 & 7 & 7 & 69 \\
\hline Contact other & & & & & & & & & \\
\hline Pharmacists & 62 & 17 & 7 & 68 & 8 & 10 & 65 & 7 & 14 \\
\hline Technicians & 57 & 15 & 11 & 60 & 14 & 9 & 62 & 12 & 9 \\
\hline Self-managemer & & & & & & & & & \\
\hline Pharmacists & 82 & 4 & 0 & 79 & 3 & 4 & 85 & I & 0 \\
\hline Technicians & 83 & 0 & 0 & 81 & 0 & 2 & 83 & 0 & 0 \\
\hline
\end{tabular}


Table S2 (Continued)

\begin{tabular}{|c|c|c|c|c|c|c|c|c|c|}
\hline & \multicolumn{3}{|c|}{ Question } & \multicolumn{3}{|c|}{ Giving information } & \multicolumn{3}{|c|}{ Counseling } \\
\hline & 0 & I & $>1$ & 0 & I & $>1$ & 0 & I & $>1$ \\
\hline \multicolumn{10}{|l|}{ Facilitators } \\
\hline Pharmacists & 83 & 1 & 2 & 61 & 6 & 19 & 85 & 1 & 0 \\
\hline Technicians & 74 & 7 & 2 & 59 & 11 & 13 & 83 & 0 & 0 \\
\hline \multicolumn{10}{|l|}{ Barriers } \\
\hline Pharmacists & 83 & 2 & I & 66 & 6 & 14 & 86 & 0 & 0 \\
\hline Technicians & 80 & 3 & 0 & 71 & 3 & 9 & 83 & 0 & 0 \\
\hline \multicolumn{10}{|l|}{ ICS, other } \\
\hline Pharmacists & 49 & 22 & 15 & 28 & 18 & 40 & 82 & 3 & I \\
\hline Technicians & 44 & 29 & 10 & 38 & 14 & 31 & 78 & 5 & 0 \\
\hline \multicolumn{10}{|c|}{ Therapeutic: non-ICS treatment } \\
\hline Pharmacists & 22 & 18 & 46 & 14 & 6 & 66 & 56 & 13 & 17 \\
\hline Technicians & 31 & 16 & 36 & 14 & 9 & 60 & 55 & 11 & 17 \\
\hline \multicolumn{10}{|l|}{ Lifestyle/social } \\
\hline \multicolumn{10}{|l|}{ Smoking } \\
\hline Pharmacists & 72 & 8 & 6 & 74 & 6 & 6 & 83 & 3 & 0 \\
\hline Technicians & 73 & 6 & 4 & 78 & 3 & 2 & 83 & 0 & 0 \\
\hline \multicolumn{10}{|l|}{ Exercise } \\
\hline Pharmacists & 83 & 2 & I & 71 & 11 & 4 & 82 & 2 & 2 \\
\hline Technicians & 80 & 3 & 0 & 79 & 2 & 2 & 82 & 0 & I \\
\hline \multicolumn{10}{|l|}{ Weight } \\
\hline Pharmacists & 86 & 0 & 0 & 86 & 0 & 0 & 86 & 0 & 0 \\
\hline Technicians & 83 & 0 & 0 & 83 & 0 & 0 & 83 & 0 & 0 \\
\hline \multicolumn{10}{|l|}{ Nutrition } \\
\hline Pharmacists & 85 & 1 & 0 & 83 & 2 & I & 85 & I & 0 \\
\hline Technicians & 83 & 0 & 0 & 81 & 2 & 0 & 83 & 0 & 0 \\
\hline \multicolumn{10}{|l|}{ Stress } \\
\hline Pharmacists & 86 & 0 & 0 & 86 & 0 & 0 & 86 & 0 & 0 \\
\hline Technicians & 83 & 0 & 0 & 81 & 2 & 0 & 83 & 0 & 0 \\
\hline \multicolumn{10}{|l|}{ Drugs } \\
\hline Pharmacists & 85 & 0 & 1 & 85 & 0 & I & 86 & 0 & 0 \\
\hline Technicians & 83 & 0 & 0 & 83 & 0 & 0 & 83 & 0 & 0 \\
\hline Influenza vaccin & & & & & & & & & \\
\hline Pharmacists & 84 & 2 & 0 & 86 & 0 & 0 & 86 & 0 & 0 \\
\hline Technicians & 83 & 0 & 0 & 82 & 0 & I & 83 & 0 & 0 \\
\hline (Living) environ & & & & & & & & & \\
\hline Pharmacists & 77 & 6 & 3 & 62 & 9 & 15 & 85 & I & 0 \\
\hline Technicians & 75 & 5 & 3 & 66 & 7 & 10 & 83 & 0 & 0 \\
\hline Social context & & & & & & & & & \\
\hline Pharmacists & 80 & 6 & 0 & 63 & 5 & 18 & 85 & I & 0 \\
\hline Technicians & 81 & 2 & 0 & 66 & 4 & 13 & 81 & I & I \\
\hline Lifestyle, other & & & & & & & & & \\
\hline Pharmacists & 78 & 5 & 3 & 73 & 7 & 6 & 84 & 2 & 0 \\
\hline Technicians & 82 & I & 0 & 73 & 7 & 3 & 83 & 0 & 0 \\
\hline Psychosocial/f & & & & & & & & & \\
\hline ICS concerns & & & & & & & & & \\
\hline Pharmacists & 85 & I & 0 & 79 & 4 & 3 & 86 & 0 & 0 \\
\hline Technicians & 83 & 0 & 0 & 82 & I & 0 & 83 & 0 & 0 \\
\hline ICS necessities & & & & & & & & & \\
\hline Pharmacists & 86 & 0 & 0 & 82 & I & 3 & 86 & 0 & 0 \\
\hline Technicians & 83 & 0 & 0 & 77 & 5 & I & 83 & 0 & 0 \\
\hline Psychosocial IC & & & & & & & & & \\
\hline Pharmacists & 65 & 17 & 4 & 65 & 8 & 13 & 84 & I & I \\
\hline Technicians & 71 & 8 & 4 & 54 & 18 & II & 83 & 0 & 0 \\
\hline Non-ICS & & & & & & & & & \\
\hline Pharmacists & 85 & 1 & 0 & 75 & 5 & 6 & 86 & 0 & 0 \\
\hline Technicians & 81 & I & I & 72 & 6 & 5 & 83 & 0 & 0 \\
\hline Other & & & & & & & & & \\
\hline Pharmacists & 66 & 11 & 9 & 0 & 0 & 86 & 85 & I & 0 \\
\hline Technicians & 79 & 4 & 0 & 0 & 0 & 83 & 83 & 0 & 0 \\
\hline
\end{tabular}

Abbreviations: COPD, chronic obstructive pulmonary disease; ICS, inhaled corticosteroids. 
Table S3 Weighted mean (SE) of behaviors of pharmacists, pharmacy technicians, and patients, controlled for visit length

\begin{tabular}{|c|c|c|c|c|}
\hline & \multicolumn{2}{|c|}{$\begin{array}{l}\text { Pharmacists' and technicians' } \\
\text { behavior }\end{array}$} & \multicolumn{2}{|l|}{ Patients' behavior } \\
\hline & Pharmacists & Technicians & Pharmacists' sessions & Technicians' sessions \\
\hline \multicolumn{5}{|l|}{ Affective part } \\
\hline Social behavior & $2.11(0.29)^{*}$ & I.22 (0.29)* & - & - \\
\hline Approval & $1.69(0.17)^{* *}$ & $1.29(0.67)^{* *}$ & - & - \\
\hline Agreements & $13.86(1.02)$ & $14.36(1.02)$ & $12.07(0.54)$ & $12.36(0.55)$ \\
\hline Verbal attention & $0.87(0.1 \mathrm{I})^{* * *}$ & $0.47(0.1 \mathrm{I})^{* * *}$ & - & - \\
\hline Shows concern or worry & $0.09(0.02)^{*}$ & $0.03(0.02)^{*}$ & - & - \\
\hline Reassurance & $1.79(0.15)$ & $1.76(0.15)$ & - & - \\
\hline Disagree & $0.05(0.01)^{*}$ & $0.02(0.01)^{*}$ & - & - \\
\hline \multicolumn{5}{|l|}{ Instrumental part } \\
\hline \multicolumn{5}{|l|}{ Process-oriented } \\
\hline Paraphrase/check for understanding & $4.06(0.35)^{*}$ & $4.76(0.35)^{*}$ & - & - \\
\hline \multicolumn{5}{|l|}{ Task-oriented } \\
\hline \multicolumn{5}{|l|}{ Question } \\
\hline \multicolumn{5}{|l|}{ Medical } \\
\hline Current health status & $0.30(0.05)^{*}$ & $0.18(0.05)^{*}$ & - & - \\
\hline Heredity & $-0.00(0.02)^{* *}$ & $0.03(0.02)^{* *}$ & - & - \\
\hline Medical, other & $0.21(0.05)^{*}$ & $0.09(0.05)^{*}$ & - & - \\
\hline \multicolumn{5}{|l|}{ ICS treatment } \\
\hline Reason for use/need & $0.12(0.03)$ & $0.08(0.03)$ & - & - \\
\hline Dosage & - & - & $0.08(0.02)^{*}$ & $0.02(0.02)^{*}$ \\
\hline Side-effects & $0.35(0.09)^{* * *}$ & $0.69(0.09)^{* * *}$ & - & - \\
\hline Self-management & $0.01(0.01)$ & $0.00(0.01)$ & - & - \\
\hline Non-ICS treatment & $0.54(0.09)$ & $0.54(0.09)$ & - & - \\
\hline \multicolumn{5}{|l|}{ Lifestyle/social } \\
\hline Social context & - & - & $0.01(0.00)$ & $0.00(0.00)$ \\
\hline Lifestyle, other & $0.03(0.01)^{*}$ & $0.00(0.01)^{*}$ & - & - \\
\hline Other & $0.10(0.02)^{* * *}$ & $0.02(0.02)^{* * *}$ & - & - \\
\hline \multicolumn{5}{|l|}{ Give information } \\
\hline \multicolumn{5}{|l|}{ Medical } \\
\hline Medical history & - & - & $1.00(0.12)^{*}$ & $0.63(0.13)^{*}$ \\
\hline Lung function test & $0.22(0.08)^{*}$ & $0.03(0.08)^{*}$ & - & - \\
\hline Explanation about asthma/COPD & $0.49(0.10)^{* * *}$ & $0.02(0.10)^{* * * *}$ & & \\
\hline \multicolumn{5}{|l|}{ ICS treatment } \\
\hline Reason for use/need & $0.24(0.04)$ & $0.16(0.04)$ & - & - \\
\hline Dosage & $0.76(0.09)^{* * *}$ & $0.39(0.09)^{* * * *}$ & $1.38(0.14)$ & $1.30(0.14)$ \\
\hline Medicinal effects & $1.67(0.15)^{* *}$ & $1.07(0.15)^{* *}$ & - & - \\
\hline Inhaler/inhalation & $6.02(0.55)$ & $6.09(0.55)$ & - & - \\
\hline Barriers & - & - & $0.19(0.04)$ & $0.09(0.04)$ \\
\hline Non-ICS treatment & $2.63(0.30)$ & $2.09(0.3 \mathrm{I})$ & - & - \\
\hline \multicolumn{5}{|l|}{ Lifestyle/social } \\
\hline (Living) environment & - & - & $0.35(0.06)^{*}$ & $0.22(0.06)^{*}$ \\
\hline Social context & $0.25(0.06)$ & $0.16(0.06)$ & - & - \\
\hline \multicolumn{5}{|l|}{ Psychosocial/feelings } \\
\hline ICS Concerns & $0.03(0.01)^{*}$ & $0.00(0.01)^{*}$ & $0.23(0.05)^{*}$ & $0.11(0.05)^{*}$ \\
\hline Other & $4.79(0.40)$ & $4.82(0.40)$ & $4.10(0.32)$ & $3.68(0.32)$ \\
\hline \multicolumn{5}{|l|}{ Counsel } \\
\hline \multicolumn{5}{|l|}{ ICS treatment } \\
\hline Adherence & $0.05(0.04)^{*}$ & $0.13(0.04)^{*}$ & - & - \\
\hline
\end{tabular}

Notes: *Significant at $P<0.05$. **Significant at $P<0.01$. ***Significant at $P<0.001$.

Abbreviations: SE, standard error; COPD, chronic obstructive pulmonary disease; ICS, inhaled corticosteroids.

Patient Preference and Adherence

\section{Publish your work in this journal}

Patient Preference and Adherence is an international, peer-reviewed, open access journal that focuses on the growing importance of patient preference and adherence throughout the therapeutic continuum. Patient satisfaction, acceptability, quality of life, compliance, persistence and their role in developing new therapeutic modalities and compounds to optimize

\section{Dovepress}

clinical outcomes for existing disease states are major areas of interest for the journal. This journal has been accepted for indexing on PubMed Central. The manuscript management system is completely online and includes a very quick and fair peer-review system, which is all easy to use. Visit http://www. dovepress.com/testimonials.php to read real quotes from published authors. 